Recepción: 20 / 04 / 2017

Aceptación: 20 / 05 / 2017

Publicación: 15 / 06 / 2017

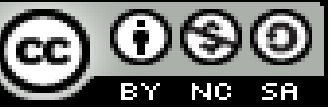

Ciencias de la Salud

\title{
Frecuencia y causas de desgarros perineales en el parto humanizado en la sala de parto
}

Frequency and causes of perineal tears in

humanized delivery in the delivery room

\section{Frequência e causas de laceração perineal no parto humanizado na sala de parto}

\author{
Yazmin de las Mercedes Granda-Barba ${ }^{\mathrm{I}}$ \\ yazmin.grandab@ug.edu.ec \\ Carlos H. Malatay-Gonzalez II \\ carlos.malatay@hotmail.com \\ Sonia B. Gómez-Vergara ${ }^{\text {III }}$ \\ sonia.gomez@hotmail.com \\ Geomayra M. Yépez-Zambrano ${ }^{\text {IV }}$ \\ geomayra.yepez@hotmail.co
}

Correspondencia: yazmin.grandab@ug.edu.ec

\footnotetext{
Magister en Nutrición; Diplomado en Docencia Superior; Obstetra; Universidad de Guayaquil, Guayaquil, Ecuador. Magister en Docencia Universitaria e Investigación Educativa; Especialista en Cirugía General; Especialista en Cirugía Oncológica; Doctor en Medicina y Cirugía; Universidad de Guayaquil, Guayaquil, Ecuador.

III. Magister en Nutrición; Diplomado en Docencia Superior; Obstetra; Universidad de Guayaquil, Guayaquil, Ecuador.

Iv. Obstetra, Universidad de Guayaquil, Guayaquil, Ecuador.
} 


\section{Resumen}

Nuestro proyecto es realizado con la finalidad de demostrar que el parto humanizado es un fundamento muy bueno para nuestras pacientes que no solo se trata del desgarro perineal sino también del trato que reciben ellas, es por eso que nuestra investigación tiene como objetivo demostrar que el parto humanizado es la mejor opción.

Esta investigación es de tipo descriptiva, prospectiva, de diseño no experimental y de corte transversal.

Los motivos de nuestra investigación son de averiguar si el parto humanizado minimiza los riesgos de desgarros; y en caso que hubiera, saber el motivo por el cual se produjeron.

Para realizar esta investigación usamos las historias clínicas de las pacientes que ingresaron a la sala de parto en periodo de expulsivo, en el tiempo estimado pudimos recopilar la información de 100 pacientes.

Pudimos obtener de este estudio los siguientes resultados:

Que de todo nuestro universo de 100 pacientes, un total de 72 pacientes no tuvieron ningún tipo de desgarro, ni lesión.

Las 28 pacientes restantes de nuestro universo sufrieron desgarros debido a las siguientes causas:

- Pacientes que no colaboraron en el momento del expulsivo un $71 \%$ que corresponde a 20 pacientes.

- Mala aplicación de la técnica de Ritgen un $11 \%$ que corresponde a 3 pacientes.

- Paciente que obtuvieron productos grandes dentro de las características normales para generarse un parto por vía vaginal un $18 \%$ que corresponde a 5 pacientes.

Con estas cifras podemos decir que el parto en libre posición o humanizado es lo mejor que podemos brindar a nuestras pacientes, para así evitar problemas a futuros de salud ginecológica.

Palabras claves: Parto humanizado; desgarro; periné; libre posición. 


\section{Abstract}

Our project is carried out with the aim of demonstrating that humanized delivery is a very good foundation for our patients, which is not only the perineal tear but also the treatment they receive. This is why our research aims to show that delivery Humanized is the best option.

This research is descriptive, prospective, non-experimental and cross-sectional design.

The reasons for our investigation are to find out if the humanized childbirth minimizes the risks of tears; And if there was, know the reason for which they occurred.

To carry out this research, we used the clinical records of the patients who entered the delivery room during the expulsive period, in the estimated time we were able to collect information from 100 patients.

We were able to obtain from this study the following results:

That of all our universe of 100 patients, a total of 72 patients did not have any type of tear, nor lesion.

The remaining 28 patients in our universe suffered from tears due to the following causes:

- Patients who did not cooperate at the time of the expulsion $71 \%$ corresponding to 20 patients.

- Poor application of the Ritgen technique $11 \%$ corresponding to 3 patients.

- Patient who obtained large products within the normal characteristics to generate a vaginal delivery $18 \%$ corresponding to 5 patients.

With these figures we can say that delivery in free position or humanized is the best we can provide our patients, to avoid future problems of gynecological health.

Key words: Humanized childbirth, tear, perineum, free position. 


\section{Resumo}

Nosso projeto é realizado, a fim de demonstrar que o parto humanizado é uma base muito boa para os nossos pacientes é a laceração perineal, mas também o tratamento que recebem deles não está sozinho, é por isso que nossa pesquisa tem como objetivo demonstrar que o parto humanizado é a melhor escolha.

Esta pesquisa é descritiva, tipo prospectivo, design não experimental e transversal.

As razões de nossa pesquisa são para determinar se o parto humanizado minimiza o risco de lágrimas; e se houver, sei por que eles ocorreram.

Para fazer esta pesquisa, usamos os registros médicos de pacientes internados em sala de parto no período expulsivo, o tempo estimado que coletar informações de 100 pacientes.

Nós fomos capazes de obter a partir deste estudo os seguintes resultados:

Que todo o nosso universo de 100 pacientes, um total de 72 pacientes não tiveram qualquer rasgo ou lesão.

Os restantes 28 doentes em nosso universo sofreu lacerações devido às seguintes razões:

- Pacientes que não cooperaram no momento da expulsivo $71 \%$, correspondendo a 20 pacientes.

- A aplicação errada da arte Ritgen $11 \%$, correspondendo a 3 pacientes.

- grandes produtos obtidos de pacientes dentro das características normais para gerar um parto vaginal $18 \%$, correspondendo a 5 pacientes.

Com estes números, podemos dizer que o nascimento ou posição livre humanizado é o melhor que podemos oferecer aos nossos pacientes, para evitar problemas futuros de saúde ginecológica.

Palavras-chave: parto humanizado, lacrimais, períneo, posição livre. 


\section{Introducción.}

"Para cambiar al mundo es preciso cambiar la forma de nacer" (1)

Parir y nacer son momentos únicos e irrepetibles de nuestras vidas. El parto es mucho más que un mero evento fisiológico donde se ponen en marcha una serie de hormonas y órganos; es probablemente el momento más trascendental, intenso y maravilloso para una mujer, es el inicio de una vida, el descubrimiento del enorme poder que tenemos dentro las mujeres; es, sin dudas, un antes y un después en la vida de esa mujer, de su pareja y de ese ser humano que nace. Y es, por sobre todas las cosas, un evento natural, saludable y seguro. (2)

La humanización del parto y nacimiento es un movimiento mundial que está obteniendo el reconocimiento cada vez de más personas en el mundo. Se basa en el respeto a los Derechos Humanos, el propósito es promover la normalidad, lo fisiológico, seguro, saludable y trascendente para el que las mujeres están innatamente preparadas. (3)

Un parto humanizado significa reconocer no intervenir ni interferir rutinariamente en este proceso natural, no habiendo una situación de riesgo evidente. (4)

Reconocer y respetar las necesidades individuales de cada mujer, pareja y el modo en que desean transcurrir esta experiencia.

Favorecer la libertad de posición y movimiento de la mujer durante el trabajo de parto (en cuclillas, en el agua o como desee).

Para disminuir la mortalidad materna y neonatal, el MSP promueve políticas y acciones de salud para la atención del parto institucional. Esto incluye el desarrollo del Parto Tradicional Humanizado (PTH) en los servicios de salud del MSP. (5)

En abril del 2016 la Asamblea Nacional del Ecuador tratará la nueva ley de parto humanizado que se regirá en el país.

Por ello hemos propuesto realizar un estudio acerca de la frecuencia de desgarros perineales en el parto humanizado a realizarse en el Hospital Especializado Mariana de Jesús; para ello se realizara un estudio prospectivo exploratorio no experimental de corte transversal, con los resultados 
obtenidos plantearemos una propuesta que conduzca a desmentir todos los mitos acerca del parto humanizado.

\section{Materiales y métodos.}

\section{Localización}

Esta investigación se la realizara en el Hospital Especializado Mariana de Jesús de la ciudad de Guayaquil, ubicado en La 27 y Rosendo Avilés.

\section{Caracterización de la zona de trabajo}

El Hospital Especializado Mariana de Jesús, es una institución que está dirigida a la atención ginecológica, obstétrica y neonatal. Sus instalaciones estas construidas con la finalidad de atender un promedio de 11.000 pacientes al año; entre control prenatal, parto, cesáreas.

Consta con servicios como: Obstetricia, Ginecología, Mastología, Clínica VIH, Pediatría, Neonatología, Anestesiología, Banco de Leche, Tamizaje neonatal, Vacunación, Hospitalización, Partos, Cesáreas, Histerectomías, Ligaduras, Legrados, EKG, Rayos X, Colposcopia, Ecografía. Consta con un número de 62 camas para hospitalización, 2 quirófanos, 1 sala de parto con 2 camas ginecológicas, 1 sala de preparto con 13 camas, 1 sala de recuperación, 1 sala de UCIN, 1 sala de preparación neonatal.

Universo y muestra

\section{Universo}

Pacientes en periodo de expulsivo, de la sala de atención de parto humanizado del Hospital Especializado Mariana de Jesús.

\section{Muestra}

Se estudiará a 100 pacientes en periodo de expulsivo en sala de parto humanizado, que serán atendidas desde Junio a de Diciembre del 2016, en el Hospital Santa Mariana de Jesús.

\section{Método de investigación}

Tipo de investigación 
La presente investigación es de tipo descriptiva porque se describe minuciosamente la investigación y de prospectiva ya que se realizó en la sala de parto en el actual año.

\section{Diseño de la investigación}

No experimental, de corte transversal.

\section{Procedimiento de la investigación}

\section{Operacionalización}

Se realizarán las historias clínicas en preparto que estén destinadas al Parto Humanizado, se observará el periodo expulsivo para constatar la presencia de desgarros.

\section{Resultados.}

Los resultados obtenidos en la recolección de datos e historias clínicas son el índice de desgarros perineales y sus causas determinadas en mujeres atendidas en el trabajo de parto en la sala de parto humanizado, en el Hospital de Especialidades Mariana de Jesús.

\begin{tabular}{|l|c|c|}
\hline \multicolumn{2}{|c|}{$\begin{array}{c}100 \text { pacientes de la sala de parto humanizado que } \\
\text { sufrieron desgarro }\end{array}$} & Porcentajes \\
\hline Pacientes sin desgarro & 72 pacientes & $72 \%$ \\
\hline Pacientes con desgarro & 28 pacientes & $28 \%$ \\
\hline
\end{tabular}

\section{Cuadro 1}

Objetivo general: Determinar la frecuencia de desgarros perineales en el parto humanizado en la sala de parto en el hospital Santa Mariana de Jesús, de junio a diciembre del 2016. 
Yazmin de las Mercedes Granda-Barba; Carlos H. Malatay-Gonzalez; Sonia B. Gómez-Vergara; Geomayra M. Yépez-

\section{PACIENTES DE LA SALADE PARTO HUMANIZADO}

pacientes sin desgarro pacientes con desgarro

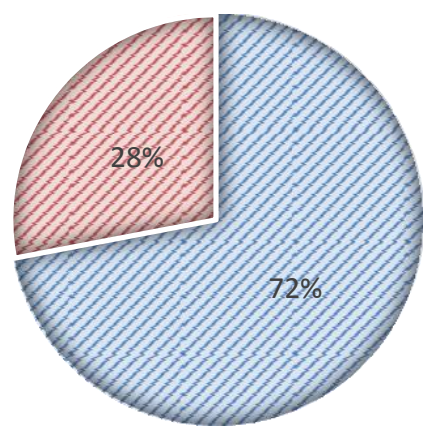

\section{Grafico 1}

Análisis: Del 100\% de las pacientes que fueron atendidas en la sala de parto humanizado pudimos constatar que el $72 \%$ de ellas no tuvieron desgarros perineales y que el $28 \%$ de las pacientes si tuvieron.

\begin{tabular}{|c|c|c|}
\hline \multicolumn{2}{|c|}{ Pacientes que no colaboran } \\
\hline Pacientes con desgarro & 28 pacientes & $58 \%$ \\
\hline $\begin{array}{c}\text { Pacientes que no } \\
\text { colaboran }\end{array}$ & 20 pacientes & $42 \%$ \\
& & \\
\hline
\end{tabular}

\section{Cuadro 2}

Objetivo específico 1: Determinar cuánto influye la colaboración de la paciente en el periodo de expulsivo para evitar los desgarros. 


\section{PACIENTES QUE NO COLABORAN}

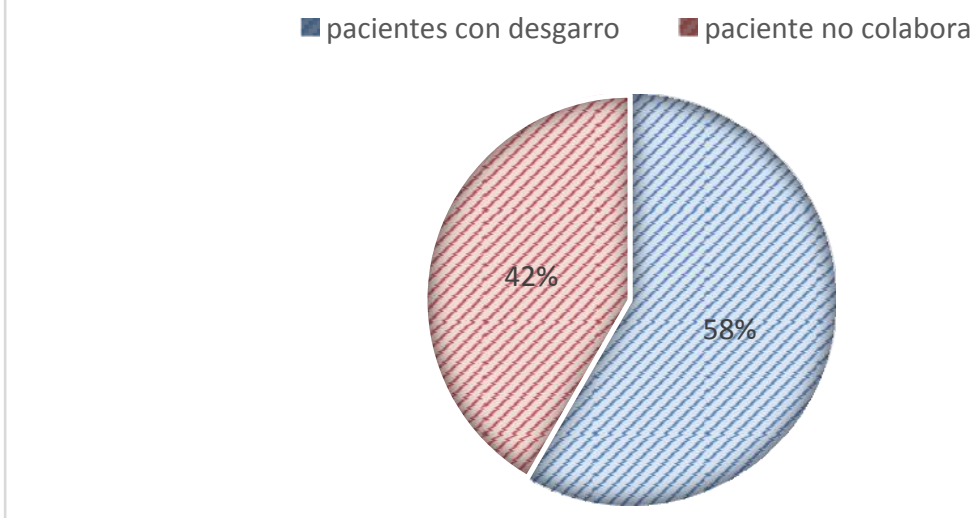

\section{Grafico 2}

Análisis: del 28\% de las pacientes que se sufrieron un desgarro perineal, en un $1 \%$ de ellas fue porque no colaboraron en el momento del expulsivo.

\begin{tabular}{|l|c|c|}
\hline \multicolumn{2}{|c|}{ Mala técnica de Ritgen } \\
\hline Pacientes con desgarro & 28 pacientes & $90 \%$ \\
\hline Mala técnica de Ritgen & 3 pacientes & $10 \%$ \\
\hline
\end{tabular}

\section{Cuadro 3}

Objetivo específico 2: definir si se utiliza una buena técnica de Ritgen para protección del periné y cuanto influye esto para evitar los desgarros. 
Yazmin de las Mercedes Granda-Barba; Carlos H. Malatay-Gonzalez; Sonia B. Gómez-Vergara; Geomayra M. Yépez-

\section{MALA TECNICA DE RITGEN}

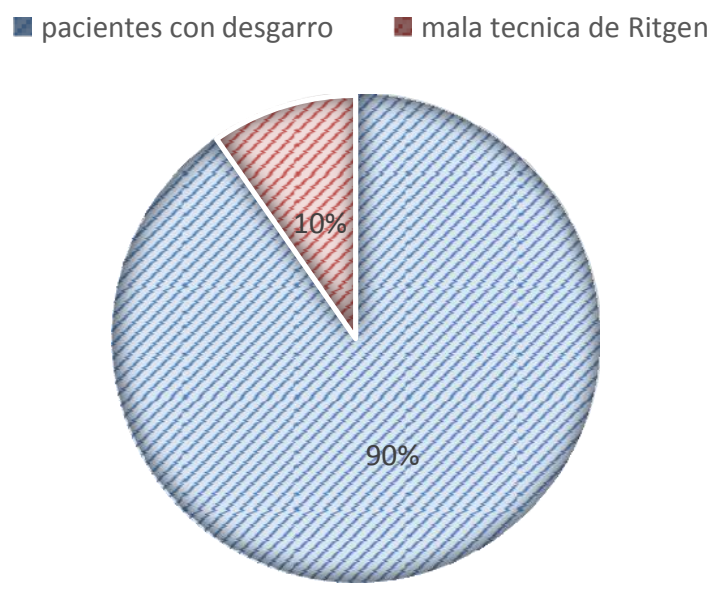

\section{Grafico 3}

Análisis: Del 28\% de las pacientes que sufrieron un desgarro el 10\% fue por una mala aplicación de la maniobre de Ritgen.

\begin{tabular}{|l|c|c|}
\hline \multicolumn{2}{|c|}{ Peso del producto } \\
\hline Pacientes con desgarro & 28 pacientes & $85 \%$ \\
& & \\
\hline Peso del producto & 5 pacientes & $15 \%$ \\
& & \\
\hline
\end{tabular}

\section{Cuadro 4}

Objetivo específico 3: determinar cuánto influye el peso del producto en los desgarros perineales. 


\section{TAMAÑO DEL PRODUCTO}

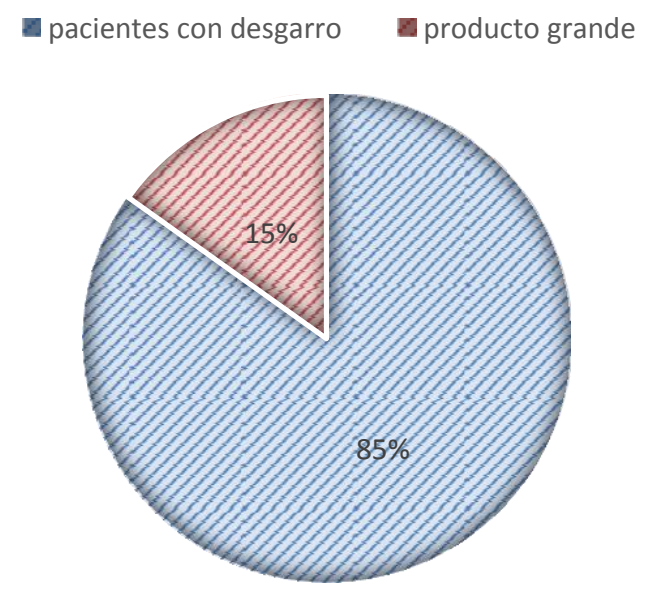

\section{Grafico 4}

Análisis: Del 28\% de las pacientes que sufrieron desgarros, el 15\% fue porque el producto era de gran tamaño, pero dentro de los parámetros normales.

\begin{tabular}{|c|c|}
\hline \multicolumn{2}{|c|}{ Causa de desgarros } \\
\hline Paciente no colabora & 20 Pacientes \\
\hline Mala técnica de Ritgen & 3 Pacientes \\
\hline Producto grande & 5 Pacientes \\
\hline
\end{tabular}

\section{Cuadro 5}

Objetivo específico 4: concluir cuál es la causa más frecuente de los desgarros perineales. 


\section{CAUSAS DE DESGARROS}

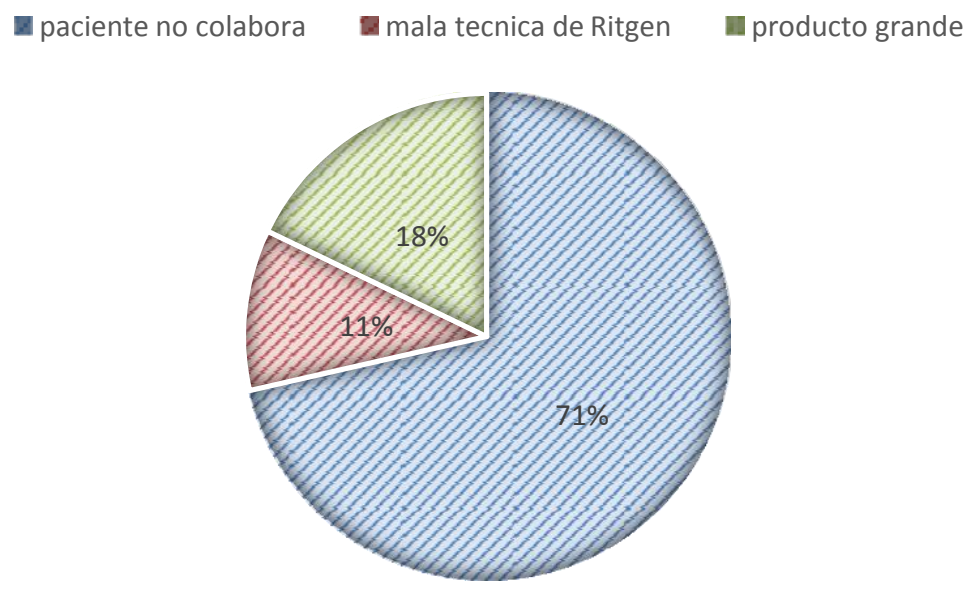

Grafico 5

Análisis: Del 28\% de las pacientes que sufrieron un desgarro podemos apreciar que el $71 \%$ es debido a que las pacientes no colaboran, el $18 \%$ es por un producto grande y el $11 \%$ es por una mala técnica de la maniobra de Ritgen

\section{Conclusiones.}

1. Después de obtener los datos de las historias clínicas realizadas y el acercamiento a las pacientes de la sala de parto humanizado del Hospital de Especialidades Mariana de Jesús podemos concluir en que:

2. Del $100 \%$ de nuestro universo solo el $28 \%$ de las pacientes sufrieron algún tipo de desgarro y el $72 \%$ de ella no lo sufrieron.

3. Del $28 \%$ de las pacientes con desgarro pudimos constatar que el $42 \%$ fue debido a que la paciente no colaboro en el momento del expulsivo.

4. Que del $28 \%$ de nuestras pacientes con desgarro el $10 \%$ fue por una mala técnica de la maniobra de Ritgen por parte de quien atendía el parto. Cabe recalcar es un porcentaje bajo.

5. Del $28 \%$ de las pacientes con desgarro el $15 \%$ fue producido por un producto grande, pero que está dentro de las medidas normales para que se produzca un parto normal.

6. Concluimos con que la mayor causa de desgarro es la poca colaboración de la paciente ya que entre las tres causas esta alcanzó un $71 \%$. 
Frecuencia y causas de desgarros perineales en el parto humanizado en la sala de parto

\section{Agradecimiento}

El agradecimiento de este proyecto va dirigido principalmente a Dios quien ha sido pilar fundamental en nuestras vidas.

A nuestra distinguida directora Obst. Delia Crespo Antepara Msc. por su apoyo incondicional en nuestro pasó por la carrera de Obstetricia y por su colaboración en los inicios de este proyecto.

A nuestra admirable tutora Obst. Yazmín Granda Barba Msc. por cada una de sus enseñanzas y que sin su ayuda y sin su conocimiento no se hubiese hecho posible la culminación de este trabajo.

A cada uno de nuestros familiares por su apoyo incondicional en especial a Edisson Mosquera, Keyller Mosquera, Cruz Zambrano, Ma. de los Ángeles Yépez y Diana Mosquera por parte de Geomayra Yépez; a Patricia Ulloa y a mis queridos hermanos de parte de Josué Planta.

\section{Bibliografía.}

1. Odent M. El nacimiento renacido Buenos Aires: Errepar; 1984.

2. Odent M. La Cientificación del amor Buenos Aires: Creavida; 2001.

3. López Villagram SM. Implemetacion de la sala de parto culturalmente adecuado en el Hospital Básico del canton Pelileo provincia del Tungurahua en el año 2013. Gerencia de Salud para el desarrollo Local. Ambato: Universidad Técnica partiocular de Loja, Área de Biología; 2014.

4. Arnau Sánchez J, Martínez Roche ME, Nicolás Vigueras MD, Bas Peña E, Morales López R, Álvarez Munárriz L. Los conceptos del parto normal, natural y humanizado. El caso del area I de salud de la región de Murcia. AIBR. Revista de Antropología Iberoamericana. 2012 ago; 7(2): p. 225-247.

5. UNICEF. Parto Intercultural Humanizado. [Online].; 2009 [cited 2014. Available from: https://www.unicef.org/ecuador/health nutrition 16853.htm. 